\title{
Chiral symmetry restoration and strong CP violation in a strong magnetic background
}

\section{Eduardo S. Fraga*}

Instituto de Física, Universidade Federal do Rio de Janeiro, Caixa Postal 68528, 21941-972, Rio de Janeiro, RJ , Brazil

E-mail: fraga@if.ufrj.br

\section{Ana Júlia Mizher}

Instituto de Física, Universidade Federal do Rio de Janeiro, Caixa Postal 68528, 21941-972, Rio de Janeiro, RJ , Brazil

E-mail: anajulia@if.ufrj.br

\begin{abstract}
Motivated by the phenomenological scenario of the chiral magnetic effect that can be possibly found in high-energy heavy ion collisions, we study the role of very intense magnetic fields and strong CP violation in the phase structure of strong interactions and, more specifically, their influence on the nature of the chiral transition. Direct implications for the dynamics of phase conversion and its time scales are briefly discussed. Our results can also be relevant in the case of the early universe.
\end{abstract}

5th International Workshop on Critical Point and Onset of Deconfinement - CPOD 2009,

June 08 - 122009

Brookhaven National Laboratory, Long Island, New York, USA

${ }^{*}$ Speaker. 


\section{Introduction and motivation}

Although topologically nontrivial configurations of the gauge fields allow for a CP-violating term in the Lagrangian of QCD [1,2], experiments indicate that its numerical coefficient, known as $\theta$, is vanishingly small, $\theta \lesssim 10^{-10}[3,4]$. Nevertheless, in spite of the fact that spontaneous breaking of $\mathrm{P}$ and $\mathrm{CP}$ were proved to be forbidden in the true vacuum of $\mathrm{QCD}$ for $\theta=0$ [5], there is still room for metastable $\mathrm{CP}$-violating states at finite temperature [6]. This provides the exciting possibility of probing the topological structure of QCD, and leads to the idea that P- and CPodd domains could be produced within the quark-gluon plasma presumably formed in high-energy heavy ion collisions [7].

The experimental signatures that were proposed to identify the presence of CP violation in certain domains within the plasma rely on charge separation of hadronic matter [8]. This asymmetry can be enhanced to the point of becoming detectable by current experiments at RHIC by the presence of a very strong magnetic background, as in the case of noncentral collisions, generating a rich phenomenon denominated chiral magnetic effect [9].

The quark-gluon plasma possibly possesses regions with nonzero winding number, $Q_{w} \neq 0$, where sphaleron transitions are induced. In the particular case of noncentral collisions a strong magnetic field is generated in the orbital angular momentum direction, perpendicular to the reaction plane. In this process, the strong magnetic field $\mathbf{B}$ restricts the quarks (all in the lowest Landau level, aligned with $\mathbf{B}$ ) to move along its direction. For a topologically nontrivial domain with e.g. $Q_{w}=-1$, left-handed quarks are converted into right-handed ones, inducing an inversion of the direction of momentum and, consequently, a net current and a charge difference are created along the direction of the magnetic field. The system is, then, P-odd.

The magnetic fields involved, although short-lived for very high energies [9], will certainly live longer for lower values of $\sqrt{s}$, and can attain enormous intensities, above those considered for magnetars [10] and comparable only to the ones believed to be present during the early stages of the universe [11]. The presence of such extreme magnetic fields coupled with strong $\mathrm{CP}$ violation raises several theoretical questions. The first one is how the QCD phase diagram is altered by the presence of a nonzero uniform magnetic field that plays the role of another "control parameter". Next one can ask where are the possible metastable CP-odd states and how "stable" they are, i.e. how long their lifetimes are. These questions are directly related to possible modifications in the nature of the phase transitions of strongly interacting matter and to the relevant time scales for phase conversion. As we have mentioned above, there is already an on-going experimental investigation that seems to show indications of the chiral magnetic effect [12]. Furthermore, pioneer lattice studies on the chiral magnetization of the non-Abelian vacuum also seem to be able to capture these effects [13].

In what follows, we discuss the effects of a strong and constant magnetic background and of $\mathrm{CP}$ violation on the chiral transition at finite temperature and vanishing chemical potential. For this purpose, we adopt the linear sigma model coupled with two flavors of quarks as our effective field theory [14], following the notation and conventions of Ref. [15]. We show that for high enough magnetic fields the chiral transition is no longer a crossover. Instead, it is turned into a first-order transition [16].

To include the effects of the presence of the axial anomaly and CP violation, we add a term 
that mimics the presence of nontrivial gauge field configurations, the 't Hooft determinant [17]. The rich vacuum structure brought about by a nonzero $\theta$ term in the action has an influence on the chiral transition, and generates a more complex picture in the analysis of the phase diagram of strong interactions [18]. Working in a mean field approximation in an extended CP-odd linear sigma model, our results can be cast in terms of condensates of the fields [19]. In this framework, three different phases occur, making the topography of extrema very rich, and allowing for the existence of metastable minima in certain situations.

\section{Effective theory for the chiral transition in a strong magnetic background}

Modifications in the vacuum of CP-symmetric QCD by the presence of a magnetic field have been investigated previously within different frameworks, mainly using effective models [20, 21, $22,23,24,25,26,27]$, especially the NJL model [28], and chiral perturbation theory [29, 30, 31], but also resorting to the quark model [32] and certain limits of QCD [33]. Most treatments have been concerned with vacuum modifications by the magnetic field, though medium effects were considered in a few cases, as e.g. in the study of the stability of quark droplets under the influence of a magnetic field at finite density and zero temperature, with nontrivial effects on the order of the chiral transition [34]. More recently, magnetic effects on the dynamical quark mass [35] and on the thermal quark-hadron transition [36], as well as magnetized chiral condensates in a holographic description of chiral symmetry breaking [37], were also considered. Recent applications of quark matter under strong magnetic fields to the physics of magnetars using the NJL model can be found in [38].

To investigate the effects of a strong magnetic background on the nature and dynamics of the chiral phase transition at finite temperature, $T$, and vanishing chemical potential, we adopt the linear sigma model coupled to two favors of quarks. Following the notation of Ref. [15], we have the lagrangian

$$
\mathscr{L}=\bar{\psi}_{f}\left[i \gamma^{\mu} \partial_{\mu}-g\left(\sigma+i \gamma_{5} \vec{\tau} \cdot \vec{\pi}\right)\right] \psi_{f}+\frac{1}{2}\left(\partial_{\mu} \sigma \partial^{\mu} \sigma+\partial_{\mu} \vec{\pi} \partial^{\mu} \vec{\pi}\right)-V(\sigma, \vec{\pi}),
$$

where $V(\sigma, \vec{\pi})=\frac{\lambda}{4}\left(\sigma^{2}+\vec{\pi}^{2}-v^{2}\right)^{2}-h \sigma$ is the self-interaction potential for the mesons, exhibiting both spontaneous and explicit breaking of chiral symmetry. The $N_{f}=2$ massive fermion fields $\psi_{f}$ represent the up and down constituent-quark fields $\psi=(u, d)$. The scalar field $\sigma$ plays the role of an approximate order parameter for the chiral transition, being an exact order parameter for massless quarks and pions. The latter are represented by the pseudoscalar field $\vec{\pi}=\left(\pi^{0}, \pi^{+}, \pi^{-}\right)$, and it is common to group together these meson fields into an $O(4)$ chiral field $\phi=(\sigma, \vec{\pi})$. In what follows, we implement a simple mean-field treatment with the customary simplifying assumptions, where quarks constitute a thermalized fluid that provides a background in which the long wavelength modes of the chiral condensate evolve. At $T=0$, the model reproduces results from chiral perturbation theory for the broken phase vacuum. In this phase, quark degrees of freedom are absent (excited only for $T>0$ ). The $\sigma$ field is heavy, $M_{\sigma} \sim 600 \mathrm{MeV}$, and treated classically. On the other hand, pions are light, and fluctuations in $\pi^{+}$and $\pi^{-}$couple to the magnetic field, $B$, as will be discussed below, whereas fluctuations in $\pi^{0}$ give a $B$-independent contribution that we ignore, for simplicity. For $T>0$, quarks are relevant (fast) degrees of freedom and chiral symmetry is approximately restored in the plasma for high enough $T$. In this case, we incorporate 
quark thermal fluctuations in the effective potential for $\sigma$, i.e. we integrate over quarks to one loop. Pions become rapidly heavy only after $T_{c}$ and their fluctuations can, in principle, matter since they couple to $B$. The parameters of the lagrangian are chosen such that the effective model reproduces correctly the phenomenology of QCD at low energies and in the vacuum, in the absence of a magnetic field. Standard integration over the fermionic degrees of freedom to one loop, using a classical approximation for the chiral field, gives the effective potential in the $\sigma$ direction $V_{\text {eff }}=V(\phi)+V_{q}(\phi)$, where $V_{q}$ represents the thermal contribution from the quarks that acquire an effective mass $M(\sigma)=g|\sigma|$. The net effect of the term $V_{q}$ is correcting the potential for the chiral field, approximately restoring chiral symmetry for a critical temperature $T_{c} \sim 150 \mathrm{MeV}$ [15].

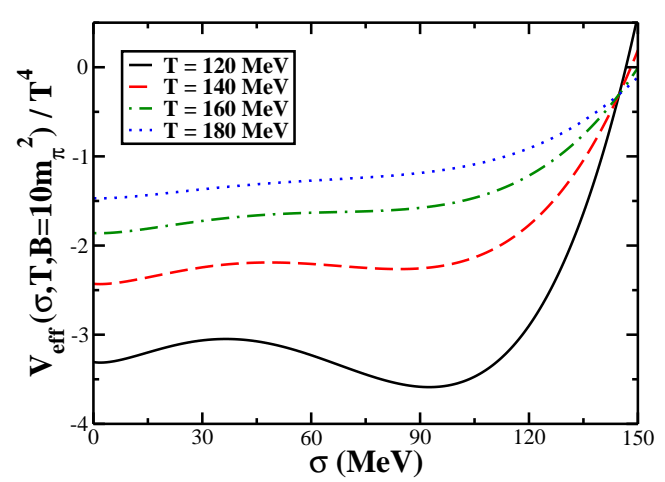

Figure 1: Evidence for a first-order chiral transition in the effective potential for $e B=10 m_{\pi}^{2}$.

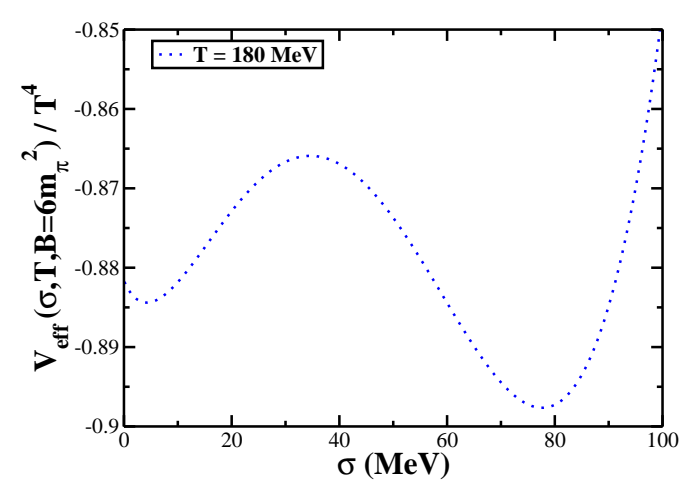

Figure 2: Zoom of the barrier for $e B=6 m_{\pi}^{2}$.

Assuming that the system is now in the presence of a strong magnetic background that is constant and homogeneous, one can compute the modified effective potential following the procedure outlined in Ref. [16]. In what follows, we simply sketch some of the main results. For definiteness, let us take the direction of the magnetic field as the $z$-direction, $\mathbf{B}=B \hat{\mathbf{z}}$. The effective potential can be generalized to this case by a simple redefinition of the dispersion relations of the fields in the presence of $\mathbf{B}$, using the minimal coupling shift in the gradient and the field equations of motion. For this purpose, it is convenient to choose the gauge such that $A^{\mu}=\left(A^{0}, \mathbf{A}\right)=(0,-B y, 0,0)$. Decomposing the fields into their Fourier modes, one arrives at eigenvalue equations which have the same form as the Schrödinger equation for a harmonic oscillator potential, whose eigenmodes correspond to the well-known Landau levels. The latter provide the new dispersion relations

$$
p_{0 n}^{2}=p_{z}^{2}+m^{2}+(2 n+1)|q| B \quad, \quad p_{0 n}^{2}=p_{z}^{2}+m^{2}+(2 n+1-\sigma)|q| B
$$

for scalars and fermions, respectively, $n$ being an integer, $q$ the electric charge, and $\sigma$ the sign of the spin. Integrals over four momenta and thermal sum-integrals are modified accordingly, yielding sums over the Landau levels.

In our effective model, the vacuum piece of the potential will be modified by the magnetic field through the coupling of the field to charged pions. To one loop, and in the limit of high $B$, 
$e B>>m_{\pi}^{2}$, one obtains (ignoring contributions independent of the condensates) [16]

$$
V_{\pi^{+}}^{V}+V_{\pi^{-}}^{V}=-\frac{2 m_{\pi}^{2} e B}{32 \pi^{2}} \log 2
$$

Thermal corrections are provided by pions and quarks. However, the pion thermal contribution as well as part of the quark thermal contribution are exponentially suppressed for high magnetic fields, as has been shown in Ref. [16]. The only part of the quark thermal piece that contributes is

$$
V_{q}^{T}=-N_{c} \frac{e B T^{2}}{2 \pi^{2}}\left[\int_{-\infty}^{+\infty} d x \ln \left(1+e^{-\sqrt{x^{2}+M_{q}^{2} / T^{2}}}\right)\right]
$$

where $N_{c}=3$ is the number of colors. Therefore, the effective potential is corrected by the contributions in (2.3) and (2.4) in the presence of a strong homogeneous magnetic background. Therefore, the presence of the magnetic field enhances the value of the chiral condensate and the depth of the broken phase minimum of the modified effective potential, a result that is in line with those found within different approaches (see, for instance, Refs. [26, 29, 31]).

Fig. 1 displays the effective potential for $e B \sim 10 m_{\pi}^{2}$ at different values of the temperature to illustrate the phenomenon of chiral symmetry restoration via a first-order transition. For RHIC top energies one expects $e B \sim 5-6 m_{\pi}^{2}$ [9]. For lower values of the field, the barrier is smaller. In Fig. 2, we show a zoom of the effective potential for $e B \sim 6 m_{\pi}^{2}$ for a temperature slightly below the critical one. This figure highlights the presence of a first-order barrier in the effective potential. For a magnetic field of the magnitude that could possibly be found in non-central high-energy heavy ion collisions, one moves from a crossover scenario to that of a weak first-order chiral transition, with a critical temperature $\sim 30 \%$ higher [16]. Although the barrier in this case is tiny, the intensity of supercooling of the system is expected to be rather large due to the smallness of nucleation rates when compared to the expansion time scales for the heavy ion scenario. Therefore, even a small barrier can keep part of the system in the false vacuum until the spinodal instability is reached and the system is abruptly torn apart. Nevertheless, since the magnetic field falls off very rapidly at RHIC top energies, we expect that even for lower values of $\sqrt{s}$ only the early-time dynamics should be affected.

As caveats, first we note that although non-central heavy ion collisions might show features of a first-order transition when contrasted to central collisions, in this comparison finite-size effects become important and have to be taken into account [39]. Second, since the magnetic field varies very rapidly in time, it can induce strong electric fields that could play a relevant role via the Schwinger mechanism.

\section{CP-odd linear sigma model}

To describe the chiral phase structure of strong interactions including CP-odd effects, we adopt an effective model that reproduces the symmetries of QCD at low energy scales, and has the appropriate degrees of freedom at each scale: the CP-odd linear sigma model coupled with two flavors of quarks. The chiral mesonic sector is built including all Lorentz invariant terms allowed by symmetry and renormalizability. Following Refs. [17, 40], one can write

$$
\mathscr{L}_{\chi}=\frac{1}{2} \operatorname{Tr}\left(\partial_{\mu} \phi^{\dagger} \partial^{\mu} \phi\right)+\frac{a}{2} \operatorname{Tr}\left(\phi^{\dagger} \phi\right)-\frac{\lambda_{1}}{4}\left[\operatorname{Tr}\left(\phi^{\dagger} \phi\right)\right]^{2}-\frac{\lambda_{2}}{4} \operatorname{Tr}\left[\left(\phi^{\dagger} \phi\right)^{2}\right]
$$




$$
+\frac{c}{2}\left[e^{i \theta} \operatorname{det}(\phi)+e^{-i \theta} \operatorname{det}\left(\phi^{\dagger}\right)\right]+\operatorname{Tr}\left[h\left(\phi+\phi^{\dagger}\right)\right] .
$$

The potential in the Lagrangian above displays both spontaneous and explicit symmetry breaking, the latter being implemented by the term $\sim h$. The strength of $\mathrm{CP}$ violation is contained in the ' $\mathrm{t}$ Hooft determinant term, which encodes the Levi-Civita structure of the axial anomaly and depends on the value of the parameter $\theta$.

Expressing the chiral field $\phi$ as

$$
\phi=\frac{1}{\sqrt{2}}(\sigma+i \eta)+\frac{1}{\sqrt{2}}\left(\vec{a}_{0}+i \vec{\pi}\right) \cdot \vec{\tau}
$$

where $\vec{\tau}$ are the generators of $S U(2)$, the Pauli matrices, the potential takes the following form (substituting the parameter $h$ by $H \equiv \sqrt{2} h$ ):

$$
\begin{aligned}
V_{\chi}= & -\frac{a}{2}\left(\sigma^{2}+\vec{\pi}^{2}+\eta^{2}+\vec{a}_{0}^{2}\right)-\frac{c}{2} \cos \theta\left(\sigma^{2}+\vec{\pi}^{2}-\eta^{2}-\vec{a}_{0}^{2}\right)+c \sin \theta\left(\sigma \eta-\vec{\pi} \cdot \vec{a}_{0}\right) \\
& -H \sigma+\frac{1}{4}\left(\lambda_{1}+\frac{\lambda_{2}}{2}\right)\left(\sigma^{2}+\eta^{2}+\vec{\pi}^{2}+\vec{a}_{0}^{2}\right)^{2}+\frac{2 \lambda_{2}}{4}\left(\sigma \vec{a}_{0}+\eta \vec{\pi}+\vec{\pi} \times \vec{a}_{0}\right)^{2}
\end{aligned}
$$

where the parameters $a, c, H, \lambda_{1}$ and $\lambda_{2}$ are fixed by vacuum properties of the mesons [19]. Quarks are coupled to the chiral fields in the same fashion as before.

Following a mean field analysis for the condensates $\langle\sigma\rangle$ and $\langle\eta\rangle$ and assuming that the remaining condensates vanish, we can compute the effective potential, which is a function of the condensates above and of the $\mathrm{CP}$ violation coefficient $\theta$, and fix all the free parameters [19]. For $\theta=0$ the model, of course, reproduces the results from the usual linear sigma model. Increasing the value of $\theta$ from zero to $\pi$, the minima of the effective potential rotate from the $\sigma$ direction almost to the $\eta$ direction, the rotation being complete only for massless quarks.

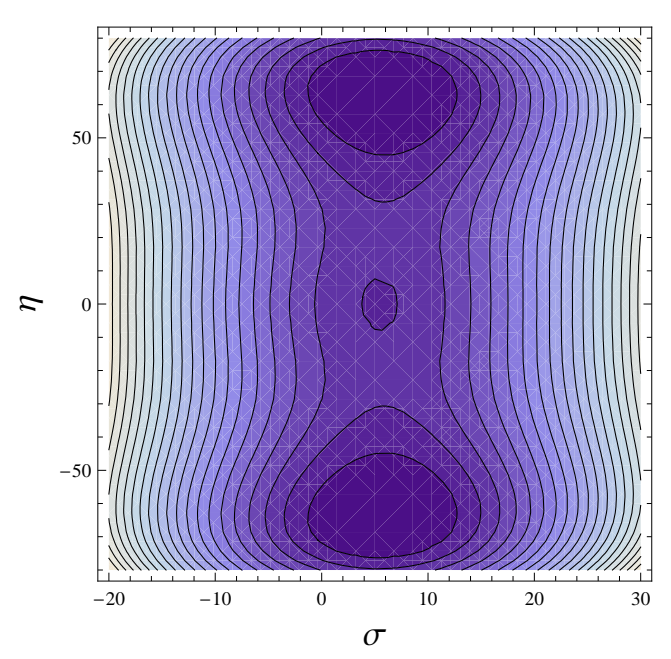

Figure 3: Contour plot of the effective potential for $\theta=\pi$ and $T=125 \mathrm{MeV}$. Numerical values are in $\mathrm{MeV}$.

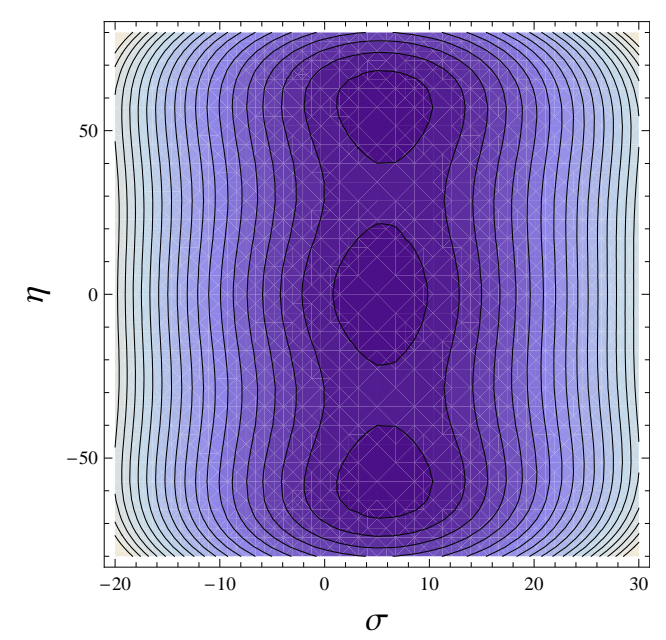

Figure 4: Contour plot of the effective potential for $\theta=\pi$ and $T=128 \mathrm{MeV}$. Numerical values are in $\mathrm{MeV}$. 
In Figs. 3 and 4 we show contour plots of the effective potential in the case of $\theta=\pi$. Increasing the temperature, the minima move towards the center, indicating chiral symmetry restoration. However, there is a clear barrier between the global minimum and the new minimum that becomes the true global minimum at high temperature (at $\eta=0$ ). In contrast to the case with $\theta=0$, this signals a first-order transition, and the possibility of metastable CP-odd states. Moreover, since the critical temperatures for the melting of $\sigma$ and $\eta$ condensates are different, three different phases are allowed in systems with $\theta$ between zero and $\pi$ : one in which both condensates are present, another where the $\eta$ condensate vanishes, and a phase where both condensates vanish [19]. This can also be illustrated by the behavior of each condensate as a function of the temperature, as shown in Fig. 5 .

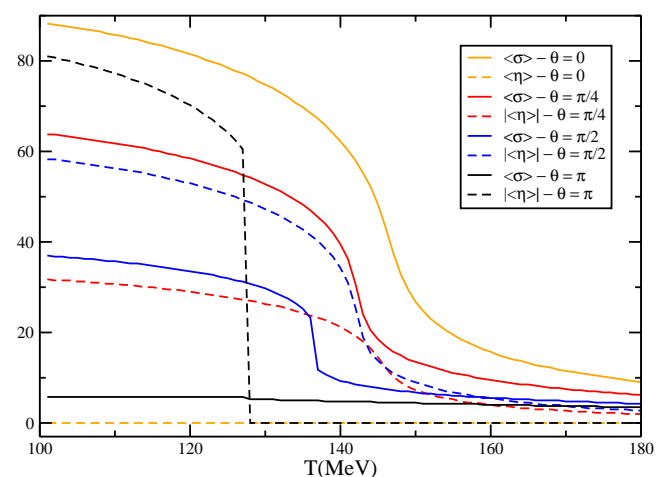

Figure 5: Absolute value of the condensates (in $\mathrm{MeV}$ ) as functions of the temperature. Full lines denote the $\sigma$ condensate and dotted lines the $\eta$ condensate.

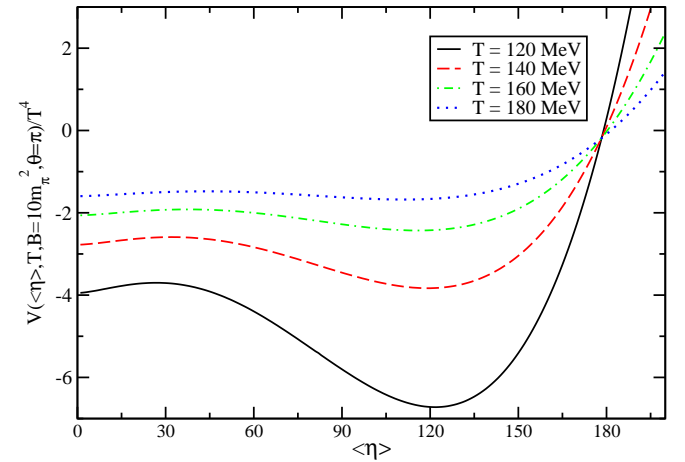

Figure 6: Effective potential normalized by the temperature for $\theta=\pi$ and $B=10 m_{\pi}^{2}$ in the direction of the $\eta$ condensate (in $\mathrm{MeV}$ ).

Following the steps of the previous section, we can incorporate the effects from a strong magnetic background on top of the CP-odd linear sigma model [19]. One then finds that the critical temperature is raised, as well as the barrier along the $\eta$ direction. Effects on the $\sigma$ field are analogous to the ones obtained in the CP-conserving linear sigma model. In Fig. 6, we illustrate this phenomenon for a magnetic field of $e B=10 m_{\pi}^{2}$ and $\theta=\pi$.

Therefore, for nonzero $\theta$ metastable $\mathrm{CP}$-violating states appear quite naturally in the $\mathrm{CP}$-odd linear sigma model. (However, they were not found in an extension of the NJL model [18]. For a discussion, see Ref. [41].) In fact, larger values of $\theta$ tend to produce a first-order chiral transition and might lead to the formation of domains (bubbles) in the plasma that exhibit CP violation [19]. This reinforces the scenario of possible metastable CP-odd states in QCD that are so relevant for the chiral magnetic effect [9]. This behavior is enhanced by the presence of a strong magnetic field, so that both effects seem to push in the same direction $[16,19]$.

\section{Final remarks}

Strong magnetic fields can modify the nature of the chiral and the deconfining transitions, opening new possibilities in the study of the phase diagram of QCD, introducing a new control 
parameter, besides temperature and baryon chemical potential, in the study of the thermodynamics of strong interactions. These high magnetic fields are also essential in the context of high-energy heavy ion collisions in generating a measurable charge asymmetry to determine the presence of CP-odd domains created by sphaleron transitions. Several relevant questions that are still not fully covered can be raised. How strong can one make these magnetic fields in current and future experiments? How long lived could they be, and how uniform, for different values of $\sqrt{s}$ ? Only the first theoretical estimates $[9,42]$ and preliminary lattice simulations [13] were performed, providing encouraging results.

On the one hand, due to the presence of a strong magnetic background, non-central heavy ion collisions might show features of a first-order transition when contrasted to central collisions. On the other hand, finite-size effects are sizable for non-central collisions [39], so that an accurate centrality dependence study seems to be necessary. This demands a thin binning of centrality and control of finite-size effects, a very difficult (but necessary) task for experimentalists in the process of data analysis, especially in the scheduled Beam Energy Scan program at RHIC-BNL.

Finally, one still needs to perform dynamical investigations within the chiral magnetic effect scenario to determine the relevant time scales and verify whether effects from the CP-odd domains survive long enough to produce measurable signatures.

\section{Acknowledgments}

We thank D. Boer, J. Boomsma, M. Chernodub, K. Fukushima, M. Stephanov and A. Zhitnitsky for fruitful discussions. E.S.F. is specially grateful to D. Kharzeev for valuable discussions and for his kind hospitality in the Nuclear Theory Group at BNL. This work was partially supported by CAPES, CNPq, FAPERJ and FUJB/UFRJ.

\section{References}

[1] A. A. Belavin, A. M. Polyakov, A. S. Shvarts and Yu. S. Tyupkin, Phys. Lett. B 59, 85 (1975).

[2] S. L. Adler, Phys. Rev. 177, 2426 (1969); J. S. Bell and R. Jackiw, Nuovo Cim. A 60, 47 (1969).

[3] K. F. Smith et al., Phys. Lett. B 234, 191 (1990); I. S. Altarev et al., Phys. Lett. B 276, 242 (1992); J. P. Jacobs, W. M. Klipstein, S. K. Lamoreaux, B. R. Heckel, and E. N. Fortson, Phys. Rev. Lett. 71, 3782 (1993).

[4] V. Baluni, Phys. Rev. D 19, 2227 (1979); R. J. Crewther, P. Di Vecchia, G. Veneziano and E. Witten, Phys. Lett. B 88, 123 (1979) [Erratum-ibid. B 91, 487 (1980)]; K. Kawarabayashi and N. Ohta, Nucl. Phys. B 175, 477 (1980); Prog. Theor. Phys. 66, 1789 (1981).

[5] C. Vafa and E. Witten, Phys. Rev. Lett. 53, 535 (1984); Nucl. Phys. B 234, 173 (1984).

[6] S. Bronoff and C. P. Korthals Altes, Phys. Lett. B 448, 85 (1999); V. Azcoiti and A. Galante, Phys. Rev. Lett. 83, 1518 (1999); T. D. Cohen, Phys. Rev. D 64, 047704 (2001); X. d. Ji, Phys. Lett. B 554, 33 (2003); M. B. Einhorn and J. Wudka, Phys. Rev. D 67, 045004 (2003); M. Creutz, Phys. Rev. Lett. 92, 201601 (2004).

[7] D. Kharzeev, R. D. Pisarski and M. H. G. Tytgat, Phys. Rev. Lett. 81, 512 (1998). 
[8] D. Kharzeev and R. D. Pisarski, Phys. Rev. D 61, 111901 (2000); K. Buckley, T. Fugleberg and A. Zhitnitsky, Phys. Rev. Lett. 84, 4814 (2000); S. A. Voloshin, Phys. Rev. C 62, 044901 (2000); S. A. Voloshin, Phys. Rev. C 70, 057901 (2004); D. Kharzeev, Phys. Lett. B 633, 260 (2006); D. Kharzeev and A. Zhitnitsky, Nucl. Phys. A 797, 67 (2007).

[9] D. E. Kharzeev, L. D. McLerran and H. J. Warringa, Nucl. Phys. A 803, 227 (2008); K. Fukushima, D. E. Kharzeev and H. J. Warringa, Phys. Rev. D 78, 074033 (2008); D. E. Kharzeev and H. J. Warringa, arXiv:0907.5007 [hep-ph]; D. E. Kharzeev, arXiv:0908.0314 [hep-ph].

[10] R. C. Duncan and C. Thompson, Astrophys. J. 392, L9 (1992); C. Thompson and R. C. Duncan, ibid. 408, 194 (1993).

[11] D. J. Schwarz, Annalen Phys. 12, 220 (2003).

[12] S. A. Voloshin and t. S. Collaboration, arXiv:0907.2213 [nucl-ex].

[13] P. V. Buividovich, M. N. Chernodub, E. V. Luschevskaya and M. I. Polikarpov, arXiv:0812.1740 [hep-lat]; arXiv:0906.0488 [hep-lat]; arXiv:0907.0494 [hep-lat].

[14] M. Gell-Mann and M. Levy, Nuovo Cim. 16, 705 (1960).

[15] O. Scavenius, A. Mocsy, I. N. Mishustin and D. H. Rischke, Phys. Rev. C 64, 045202 (2001); O. Scavenius, A. Dumitru, E. S. Fraga, J. T. Lenaghan and A. D. Jackson, Phys. Rev. D 63, 116003 (2001).

[16] E. S. Fraga and A. J. Mizher, Phys. Rev. D 78, 025016 (2008); Nucl. Phys. A 820, 103C (2009).

[17] G. 't Hooft, Phys. Rept. 142, 357 (1986).

[18] D. Boer and J. K. Boomsma, Phys. Rev. D 78, 054027 (2008).

[19] A. J. Mizher and E. S. Fraga, Nucl. Phys. A 820, 247C (2009); arXiv:0810.5162 [hep-ph].

[20] S. P. Klevansky and R. H. Lemmer, Phys. Rev. D 39, 3478 (1989).

[21] V. P. Gusynin, V. A. Miransky and I. A. Shovkovy, Phys. Lett. B 349, 477 (1995); V. P. Gusynin, V. A. Miransky and I. A. Shovkovy, Nucl. Phys. B 462, 249 (1996).

[22] A. Y. Babansky, E. V. Gorbar and G. V. Shchepanyuk, Phys. Lett. B 419, 272 (1998).

[23] K. G. Klimenko, arXiv:hep-ph/9809218.

[24] G. W. Semenoff, I. A. Shovkovy and L. C. R. Wijewardhana, Phys. Rev. D 60, 105024 (1999).

[25] A. Goyal and M. Dahiya, Phys. Rev. D 62, 025022 (2000).

[26] B. Hiller, A. A. Osipov, A. H. Blin and J. da Providencia, SIGMA 4, 024 (2008).

[27] E. Rojas, A. Ayala, A. Bashir and A. Raya, Phys. Rev. D 77, 093004 (2008).

[28] S. P. Klevansky, Rev. Mod. Phys. 64, 649 (1992).

[29] I. A. Shushpanov and A. V. Smilga, Phys. Lett. B 402, 351 (1997).

[30] N. O. Agasian and I. A. Shushpanov, Phys. Lett. B 472, 143 (2000).

[31] T. D. Cohen, D. A. McGady and E. S. Werbos, Phys. Rev. C 76, 055201 (2007).

[32] D. Kabat, K. M. Lee and E. Weinberg, Phys. Rev. D 66, 014004 (2002).

[33] V. A. Miransky and I. A. Shovkovy, Phys. Rev. D 66, 045006 (2002).

[34] D. Ebert and K. G. Klimenko, Nucl. Phys. A 728, 203 (2003). 
[35] K. G. Klimenko and V. C. Zhukovsky, Phys. Lett. B 665, 352 (2008).

[36] N. O. Agasian and S. M. Fedorov, Phys. Lett. B 663, 445 (2008).

[37] A. Rebhan, A. Schmitt and S. A. Stricker, JHEP 0905, 084 (2009).

[38] D. P. Menezes, M. Benghi Pinto, S. S. Avancini, A. Perez Martinez and C. Providencia, Phys. Rev. C 79, 035807 (2009); D. P. Menezes, M. B. Pinto, S. S. Avancini and C. Providencia, arXiv:0907.2607 [nucl-th].

[39] L. F. Palhares, E. S. Fraga and T. Kodama, arXiv:0904.4830 [nucl-th].

[40] C. Rosenzweig, J. Schechter and C. G. Trahern, Phys. Rev. D 21, 3388 (1980); R. D. Pisarski and F. Wilczek, Phys. Rev. D 29, 338 (1984); J. T. Lenaghan, D. H. Rischke and J. Schaffner-Bielich, Phys. Rev. D 62, 085008 (2000); D. Roder, J. Ruppert and D. H. Rischke, Phys. Rev. D 68, 016003 (2003); Nucl. Phys. A 775, 127 (2006).

[41] J. K. Boomsma and D. Boer, arXiv:0905.4660 [hep-ph].

[42] V. Skokov, A. Illarionov and V. Toneev, arXiv:0907.1396 [nucl-th]. 